\title{
Appearance of Saturn's F ring azimuthal channels for the anti-alignment configuration between the ring and Prometheus
}

\author{
Carlos E. Chavez ${ }^{\mathrm{a}}$,
}

\author{
anstituto de Astronomia UNAM sede Ensenada, Km. 103 carretera
}

Tijuana-Ensenada, c.p. 22860, Ensenada, Baja California, Mexico

Number of pages: 9

Number of tables: 1

Number of figures: 6 
Proposed Running Head:

Appearance of $\mathrm{F}$ ring azimuthal channels at anti-alignment

Please send Editorial Correspondence to:

Carlos E. Chavez

Instituto de Astronomia UNAM sede Ensenada

Km. 103 carretera Tijuana-Ensenada

Ensenada, Baja California, c.p. 22860, Mexico.

Email: carlosepech@astrosen.unam.mx

Phone: (0052) 6461744593 ext. 326

Fax: (0052) 6461744607 


\section{ABSTRACT}

In this article we explore the aspect of the $\mathrm{F}$ ring with respect to the antialignment configuration between the ring and Prometheus. We focus our attention on the shape of the F ring's azimuthal channels which were first reported by Porco et al. (2005) and numerically explored by Murray et al. (2005), who found excellent agreement between Cassini's ISS reprojected images and their numerical model via a direct comparison. We find that for anti-alignment the channels are wider and go deeper inside the ring material. From our numerical model we find a new feature, an island in the middle of the channel. This island is made up of the particles that have been perturbed the most by Prometheus and only appears when this satellite is close to apoapsis. In addition, plots of the anti-alignment configuration for different orbital stages of Prometheus are obtained and discussed here.

Keywords: PLANETARY RINGS; SATURN, RINGS; SATURN, SATELLITES 


\section{Introduction}

Since the azimuthal channels were first discovered with the Cassini spacecraft in July 2004 (Porco et al. (2005)), these features have captured the imagination of scientist involved in the Cassini mision. These channels have appeared 28 times in the imaging diary and appeared in one press release in the ciclops website (http://www.ciclops.org) as counted on June 1, 2008. Numerical simulations of the $\mathrm{F}$ ring have shown the possibility that azimuthal channels may form (Showalter and Burns (1982); Giuliatti Winter et al. (2000)) . But it was not until the Cassini spacecraft arrived, that this was confirmed (Porco et al. (2006)). The available initial conditions obtained from these images have permitted us to make a direct comparison between Cassini's ISS images and the numerical simulations (Murray et al. (2005)) for the first time. The dynamical complexity of the channels is evident when we see how their aspect changes over time, as a function of Prometheus orbit (Murray and Giuliatti Winter (1996), Giuliatti Winter et al. (2000) and Murray et al. (2005)). The observed changing angle is believed to be due to Keplerian shear (Murray et al. (2005)). Here we study the anti-alignment configuration between the F ring and Prometheus and the consequences for the azimuthal channels using numerical full integrations.

\section{Methods}

Possible complications that our treatment ignores, include (i) The gravitational influence of other satellites (Prometheus is without doubt the origin of the channels, as found by Murray et al. (2005)). (ii) The oblateness of Saturn and therefore its precessional effect on the orbits of the ring particles and Prometheus (mutual precession is only $0.057^{\circ} d^{-1}$, for the time scales involved here and it can be neglected). (iii) The effect of relatively large objects within the F ring core and therefore self-gravity. (iv) Collisions between $\mathrm{F}$ ring particles (this is a standard assumption when modeling the $\mathrm{F}$ ring as it was supposed in Murray et al. (2005), Giuliatti Winter et al. (2000), Murray et al. (1997), etc.).

The approximate date for the anti-alignment configuration has been calculated using recently published ephemerides. Jacobson et al. (2008) Table 5 was used for Prometheus and the solution of Bosh et al. (2002) for the F ring. We proceeded as follows: First we took an arbitrary initial orbital configuration, here we used the Cassini's Saturn Orbital Insertion (hereafter SOI) date as the initial date. This date corresponds to UT 04:48:00 on July 1, 2004. For that orbital configuration, we calculated the minimum distance between Prometheus and the $\mathrm{F}$ ring by dividing the orbit in 1000 points (which gives 
us an error in the calculation of the order of $0.36^{\circ}$ ). Then we use precessing ellipses to evolve the orbits, with that we obtain a new orbital configuration. Then we repeat the calculation of the minimum distance, and so on. The minimum distance between Prometheus and the F ring core is calculated over a time span of 44 years with a time step of 1 day.

The values of the orbital elements obtained by this method are shown in Table 1. For convenience we used the orbital elements in the reference frame in which $\widetilde{\omega}=\Omega=0$ for Prometheus. This means that in this reference frame $\widetilde{\omega}=179.8648^{\circ}$ and $\Omega=271.3077^{\circ}$ for the ring.

Figure 1 shows how the minimum distance between Prometheus and the F ring core changes over time. Starting from epoch JD 2453187.7 (corresponding to UT 04:48:00 on July 1, 2004 which is the SOI date) and finishing in epoch JD 2467787.7 (corresponding to UT 08:17:49.3 on August 27, 2027). Then the first minimum on the plot corresponds to the approximated date of the next anti-alignment event, shown in Fig. 1 as a dotted line. This date has been found to be epoch JD 2455156.5 (corresponding to UT 4:54:24 on November 21,2009 ) and the corresponding minimum distance is $180.9 \mathrm{~km}$. Since there are no sharp or abrupt changes in the plot (the shape is very smooth when approaching to minimum), the systems shows a soft transition to the antialignment configuration. This means that the shape of the channels should not change substantially around anti-alignment. In Fig. 1]we are showing dates one year before and one year after the encounter, shown here as two solid vertical lines (one at the left and one at the right of the minimum, respectively). In Fig. 1. the difference in distance between the minimum distance at anti-alignment and the line at the left is $10.8 \mathrm{~km}$, and for the one at the right is $11.3 \mathrm{~km}$. These distances are very small compared to $472.5 \mathrm{~km}$, which is the minimum distance at SOI. These dates are relevant since the Cassini spacecraft extended mission will end in July 1, 2010.

Once per orbit during this anti-alignment Prometheus will be at apoapsis and the ring particles will be at periapsis. This configuration is studied here numerically, and the possible appearance of the $\mathrm{F}$ ring for such a configuration is predicted. Much progress has been made in understanding the nature of the F ring's strands', it was found that these strands have spiral shapes (Charnoz et al. (2005)). More recently, it was found that small moonlets could be the origin of the strands (Murray et al. (2008)). Despite all these advances in understanding, it is still not possible to predict the number and location of strands in the $\mathrm{F}$ ring at a given time and at a given longitude. Therefore, it was decided not to include strands in the numerical simulation, except the core and the background sheet (we are using the names of each feature as it appears in Murray et al. (2005)). 


\section{Results}

An integration of the full equations was performed for the anti-alignment configuration, with 100,000 test particles (50,000 on each strand) using the Runge-Kutta-Nyström RKN 12(10) 17M of Brankin et al. (1989) (using the higher-order version). Numerical integrations were performed in a Saturnocentric reference frame, in which the $x-y$ plane corresponds to the equatorial plane of Saturn. As is conventionally assumed, the direction of the $x$-axis points to the ascending node of Saturn's equator on the Earth's mean equator of J2000 (2451545.0 JED). The results of the integration for the anti-alignment configuration are shown in Fig. 2 and 3 (these plots can be compared with those obtained in Murray et al. (2005) Fig. 2 a to f). The perturbation is more extreme in this case; the streamers from previous encounters are present and are more prominent than the Murray et al. (2005) plots. The positions of Prometheus and all the test particles are shown in the rotating reference frame with Prometheus' mean motion.

We show in Fig. 2 and 3 eight snapshots of the system for different orbital stages. The total time between the first plot and the last is one orbital period of Prometheus (that is 14.7462 hours), which is the natural period of time to be used since Prometheus is the cause of these features. The azimuthal channels that have been formed in previous Prometheus' orbits are clearly visible here. The structure and physical appearance of the channels are clearly time-dependent. These distortions are located in the inner part of the ring since Prometheus has a stronger influence here.

Figure 2 a (mean anomaly $M=0^{\circ}$ ) shows a ring with no channels in it, the streamers of material from previous passages are visible and the perturbations in the core strand are at their maximum extension, these streamers were first reported in Murray et al. (2005). Figure 2 b $\left(M=45^{\circ}\right)$ shows how channels start to open again, the streamers of material are still there but much smaller than in the previous plot and the perturbations in the core strand are present. Figure 2 c $\left(M=90^{\circ}\right)$ shows the channels, where the streamers of material have disappeared and the perturbations in the core strand have decreased substantially. Figure 2 d $\left(M=135^{\circ}\right)$ still shows the channels, Prometheus is getting embedded into the ring material but the perturbations in the core strand are barely visible at this point. Figure 3 e $\left(M=180^{\circ}\right)$ shows the channels at their maximum width, Prometheus is at its closest approach to the ring's core and the perturbations in the core strand are not visible. Figure $3 \mathbf{f}$ $\left(M=225^{\circ}\right)$ shows the channels, the streamers of material have now appeared again, the perturbations in the core strand are present too, and Prometheus is still embedded in the ring material. Finally, Fig. $3 \mathbf{g}\left(M=270^{\circ}\right)$ shows a configuration very similar to Fig. $3 \mathbf{h}\left(M=315^{\circ}\right)$, except that in Fig. $3 \mathbf{g}$ the channels are still present. 
It is important to notice that when Prometheus is close to apoapsis a new feature is visible in the middle of the channel for this configuration, as seen in Fig. 3 e. In Fig. 4 we are zooming on the region in which this new island of test particles appears. From our numerical results the island is visible when Prometheus is between the mean anomaly $M=144.0^{\circ}$ to $M=201.0^{\circ}$ (that is for $\sim 60.0^{\circ}$ or 2.4577 hours).

In both Figs. 2 and 3 a triangle is used to show the position of Prometheus' center, the true-scale of Prometheus is shown, as a elongated ellipse, in the left-bottom corner of Figs. 2a and $3 \mathbf{e}$.

The code utilized is able to monitor the number of collisions of ring particles with a spherical Prometheus, $50 \mathrm{~km}$ in radius ( the same value that was reported in Murray et al. (2005)) and we found that only $0.6 \%$ of the total number of test particles included in the background sheet collided with Prometheus per orbit (and none of the particles of the core strand). The relevance of this result is that it finally rules out collisions with Prometheus as the origin of the azimuthal channels. Here we are including a section of the ring that covers $24.0^{\circ}$ in mean longitude (from $\lambda=-7^{\circ}$ to $\lambda=17.0^{\circ}$ ). This longitude span was chosen because Prometheus would then encounter the $\mathrm{F}$ ring five times in the course of the integration. From here, and using the geometry of the ellipse, it is possible to find the area of this section of the ring, we find $A_{\text {sect }}=4.07045 \times 10^{7} \mathrm{~km}^{2}$, which means a surface density for the ring of $\sigma=0.00122836$ particles per $\mathrm{km}^{2}$. The total area of the ring is given by $A_{\text {total }}=6.1673 \times 10^{8} \mathrm{~km}^{2}$. That means that the percentage of the total number of particles removed, for the whole ring, is $0.038 \%$ per orbit, if we assume that this collisional rate continues steadily, and that the particles that collide with Prometheus stick to its surface, then all the particles of the background sheet should be removed within $\sim 4.41$ years. This is just a lower value since this percentage only is this high when Prometheus is at anti-alignemnt. As reported in Murray et al. (2005) there were not detected collisions for the SOI configuration (which is only 5 years apart from anti-alignment). More research needs to be done to find better estimates. A detailed discussion about the collisions and the physics of the collisions will be published soon Chavez (2009) in preparation.

We now obtain plots of radial distance versus longitude for this configuration in order to have a better idea of the appearance of the ring for this configuration. Fig. [5] shows the result of our numerical model for Prometheus' at amean anomaly of $M=180.0^{\circ}$. Here Prometheus is exactly at apoapsis and the $\mathrm{F}$ ring at periapsis, indicating that they are at their closest distance possible. As expected, the channels, are more prominent and go deeper into the ring material for this configuration, than the ones reported at SOI. The islands are clearly visible in the middle of the channels from previous encounters. The core strand seems to be unperturbed at this stage of the orbit. Fig. [6 shows the 
same numerical model, but Prometheus' mean anomaly is $M=0.0^{\circ}$, which corresponds to Prometheus periapsis and F ring aposapsis. The streamers from previous encounters are visible in the inner edge of the background sheet, and as expected streamers are more prominent to those observed in the SOI configuration. The gravitational perturbations in the core are easily seen in this plot.

\section{Summary}

The anti-alignment configuration between Prometheus and the F ring is studied here, with special emphasis in the aspect of the channels. The approximated date for such an event is November 21, 2009. At this configuration the orbit of Prometheus (which is the inner object) is at its apoapsis while the ring (the outer object) is at its periapsis, and so their distance is at its minimum.

We found that the channels for this configuration go deeper in the ring and are wider compared to the ones reported for SOI in Murray et al. (2005). We discovered a new feature which may be relevant to the F ring's structure: We called it the "island" since it resembles an islet in the middle of the channel. Every time Prometheus gets close to apoapsis $\left( \pm 30^{\circ}\right.$ from it), this new feature appears. This is a prediction of our model and should be visible when the system is in anti-alignment. The number of collisions between Prometheus and the $\mathrm{F}$ ring particles was monitored. Even for this configuration the number of collisions is quite low, only $0.038 \%$ of the particles collided with Prometheus per orbit. Thus these features are created by the gravitational perturbations of Prometheus and not by collisions, confirming Murray et al. (2005) results for the case of anti-alignment. The conclusion is that the particles are only temporarily pulled away and then drift back into the ring one orbital period of Prometheus' later. The Cassini spacecraft should be able to see these predicted features in its extended mission that ends in July 1, 2010.

\section{Future work}

The detailed physics behind the channel's formation needs to be studied. Similarly, the width of the channels as a function of the time needs to be explored. A comprehensive study of the collisions between Prometheus and the F ring particles needs to be done as well. 


\section{Acknowledgements}

I would like to thank to C. D. Murray for his helpful suggestions and criticisms. Additionally, I would like to thank to M. W. Evans and Kevin Beurle for instructive discussions. I would like thank to my reviewers A. S. Bosh and S. Charnoz for their helpful comments and suggestions. I thank Luis Aguilar for giving me the chance to develope this research and for all his comments and corrections. I thank David M. Clark for his comments and corrections. Lastly, I am grateful to IA-UNAM for their financial support.

\section{References}

A. S. Bosh, C. B. Olkin, R. G. French, and P. D. Nicholson. Saturn's F Ring: Kinematics and Particle Sizes from Stellar Occultation Studies. Icarus, 157: 57-75, May 2002.

R.W. Brankin, I. Gladwell, J.R. Dormand, P. J. Prince, and W. L. Seward. A Runge-Kutta-Nystrom Code. ACM Trans Math Soft, 15:31-40, June 1989.

S. Charnoz, C. C. Porco, E. Déau, A. Brahic, J. N. Spitale, G. Bacques, and K. Baillie. Cassini Discovers a Kinematic Spiral Ring Around Saturn. Science, 310:1300-1304, November 2005.

S. M. Giuliatti Winter, C. D. Murray, and M. Gordon. Perturbations to Saturn's F-ring strands at their closest approach to Prometheus. Planetary and Space Science, 48:817-827, August 2000.

R. A. Jacobson, J. Spitale, C. C. Porco, K. Beurle, N. J. Cooper, M. W. Evans, and C. D. Murray. Revised Orbits of Saturn's Small Inner Satellites. The Astronomical Journal, 135:261-263, January 2008.

C. D. Murray and S. M. Giuliatti Winter. Periodic collisions between the moon Prometheus and Saturn's F ring. Nature, 380:139-141, 1996.

C. D. Murray, M. K. Gordon, and S. M. Giuliatti Winter. Unraveling the Strands of Saturn's F Ring. Icarus, 129:304-316, October 1997.

C. D. Murray, C. Chavez, K. Beurle, N. Cooper, M. W. Evans, J. A. Burns, and C. C. Porco. How Prometheus creates structure in Saturn's F ring. Nature, 437:1326-1329, October 2005.

C. D. Murray, K. Beurle, N. J. Cooper, M. W. Evans, G. A. Williams, and S. Charnoz. The determination of the structure of Saturn's F ring by nearby moonlets. Nature, 453:739-744, June 2008.

C. C. Porco, E. Baker, J. Barbara, K. Beurle, A. Brahic, J. A. Burns, S. Charnoz, N. Cooper, D. D. Dawson, A. D. Del Genio, T. Denk, L. Dones, U. Dyudina, M. W. Evans, B. Giese, K. Grazier, P. Helfenstein, A. P. Ingersoll, R. A. Jacobson, T. V. Johnson, A. McEwen, C. D. Murray, G. Neukum, W. M. Owen, J. Perry, T. Roatsch, J. Spitale, S. Squyres, P. Thomas, M. Tiscareno, E. Turtle, A. R. Vasavada, J. Veverka, R. Wagner, and 
R. West. Cassini Imaging Science: Initial Results on Saturn's Rings and Small Satellites. Science, 307:1226-1236, February 2005.

C. C. Porco, J. W. Weiss, P. C. Thomas, D. C. Richardson, R. A. Jacobson, and J. Spitale. Physical Characteristics and Possible Accretionary Origins for Saturn's Small Satellites. In S. Mackwell and E. Stansbery, editors, 37th Annual Lunar and Planetary Science Conference, volume 37 of Lunar and Planetary Institute Conference Abstracts, pages 2289-+, March 2006.

M. R. Showalter and J. A. Burns. A numerical study of Saturn's F-ring. Icarus, 52:526-544, December 1982. 
Table 1

Initial Orbital Elements for Prometheus and the F ring at the approximated date for the anti-alignment configuration, corresponding to UT 4:54:24 on November 21, 2009. These were acquired using precessing ellipses, and the orbital elements reported in Jacobson et al. (2008) for Prometheus and Bosh et al. (2002) for the F ring.

\begin{tabular}{|c|c|c|}
\hline Elements & Prometheus & F ring \\
\hline$a(k m)$ & 139380.0 & 140223.7 \\
\hline$e$ & 0.0022 & 0.00254 \\
\hline$\left.i^{\circ}\right)$ & 0.008 & 0.0065 \\
\hline$\widetilde{\omega}\left(^{\circ}\right)$ & 236.2021 & 56.0669 \\
\hline$\Omega\left(^{\circ}\right)$ & 117.9717 & 29.2794 \\
\hline$\lambda\left(^{\circ}\right)$ & 77.5482 & - \\
\hline$n\left(^{\circ} /\right.$ day $)$ & 587.2852370 & - \\
\hline
\end{tabular}




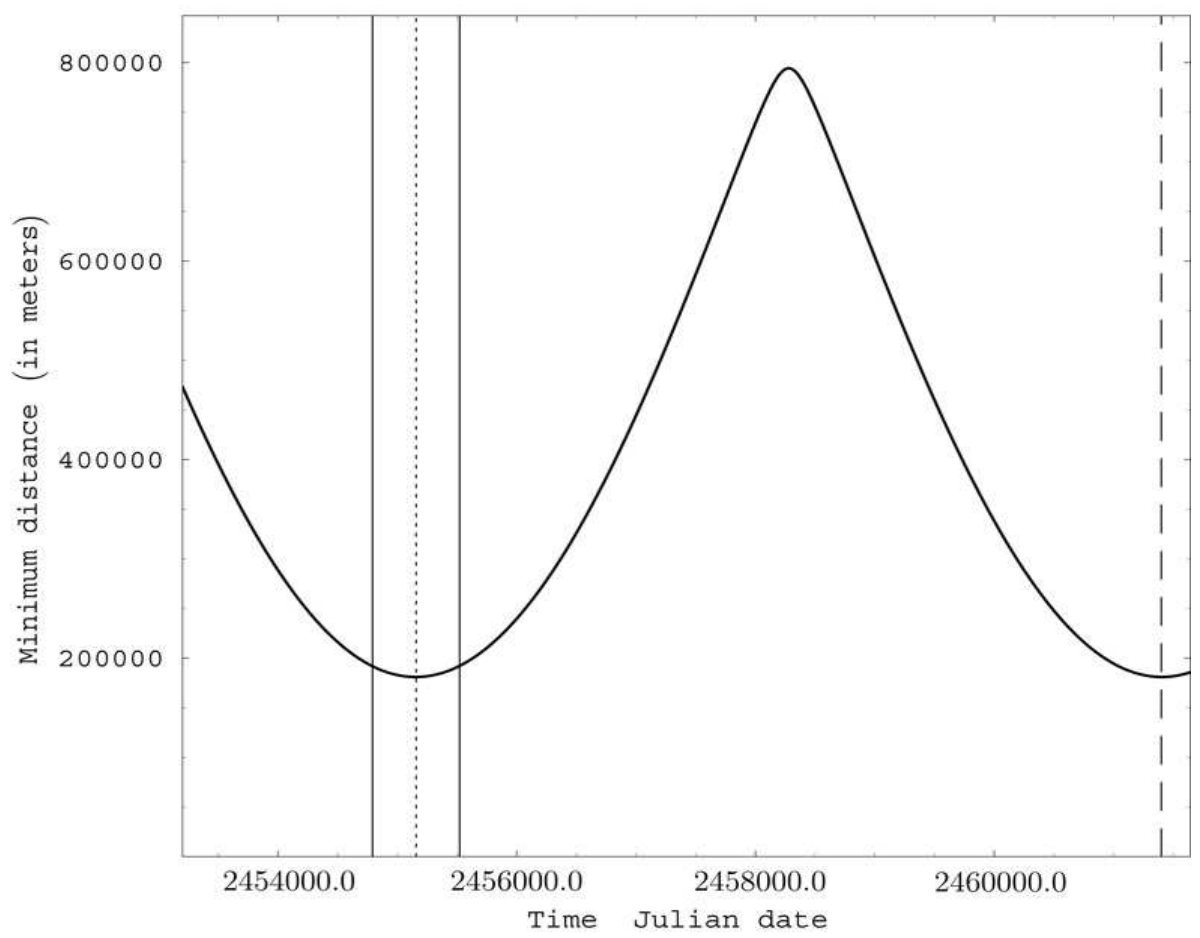

Fig. 1. The minimum distance between Prometheus and the F ring core is shown here (in meters) versus time (in Julian Date). The dotted and dashed vertical lines correspond to the next anti-alignment (November 21, 2009) and the one after it (December 24, 2026), respectively. The two vertical solid lines, one at the left and the one at the right of the dotted line, correspond to a date one year earlier and after the first anti-alignment, respectively. 

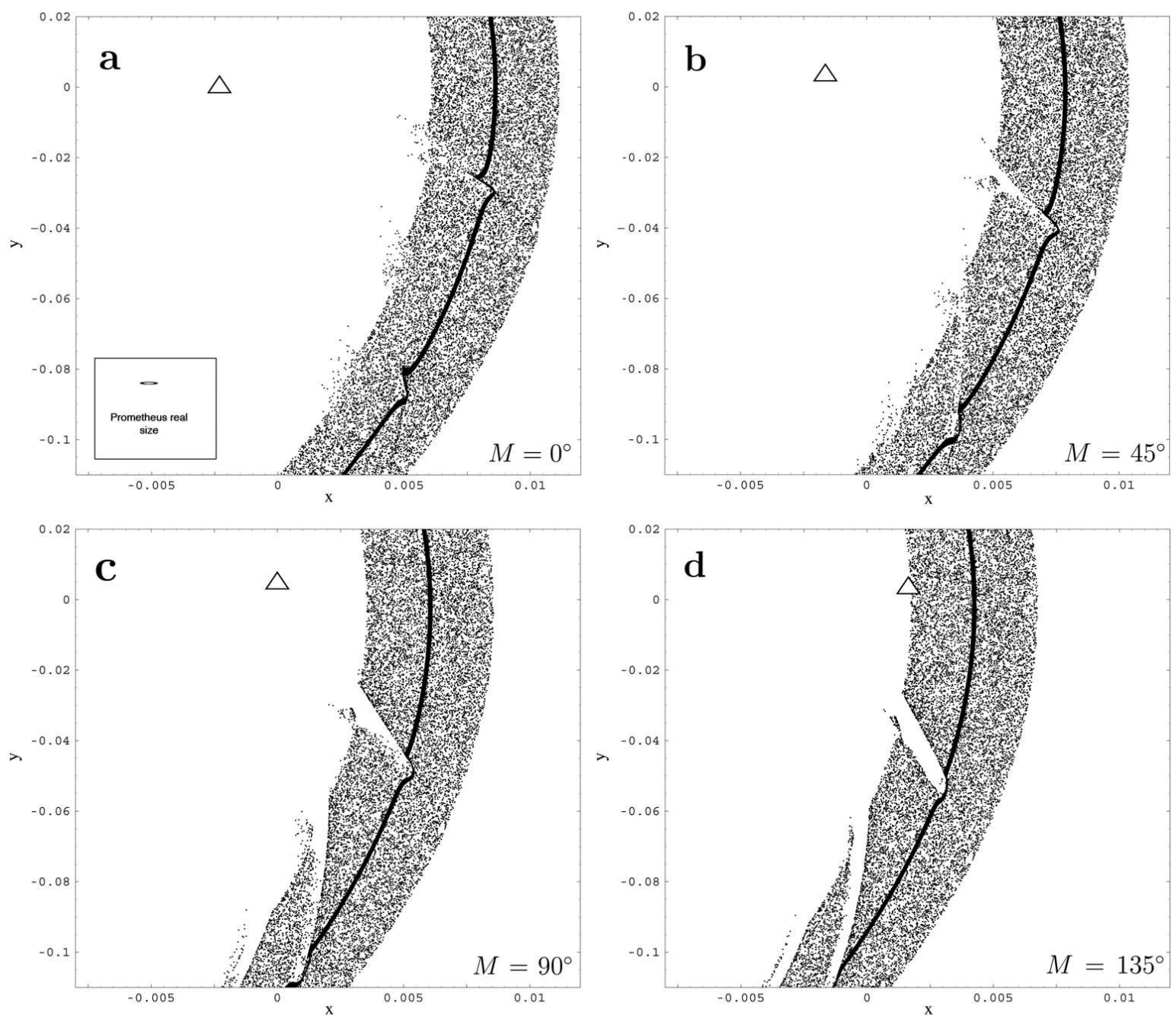

Fig. 2. First four snapshots of the encounter between Prometheus and the F ring. Prometheus is represented as a triangle $(\triangle)$ and it is used to denote the position of the center of Prometheus, the true-scale of Prometheus is shown, as a elongated ellipse, in the left-bottom corner of Fig. 2 a. These plots are in the reference frame that rotates with Prometheus mean motion. The mean anomaly $M$ for each of the plots is shown in the right bottom part of the plot. We observe that the ring is composed by two components: The core strand (at the center of the ring) and the background sheet (the less dense component that surrounds the core). 

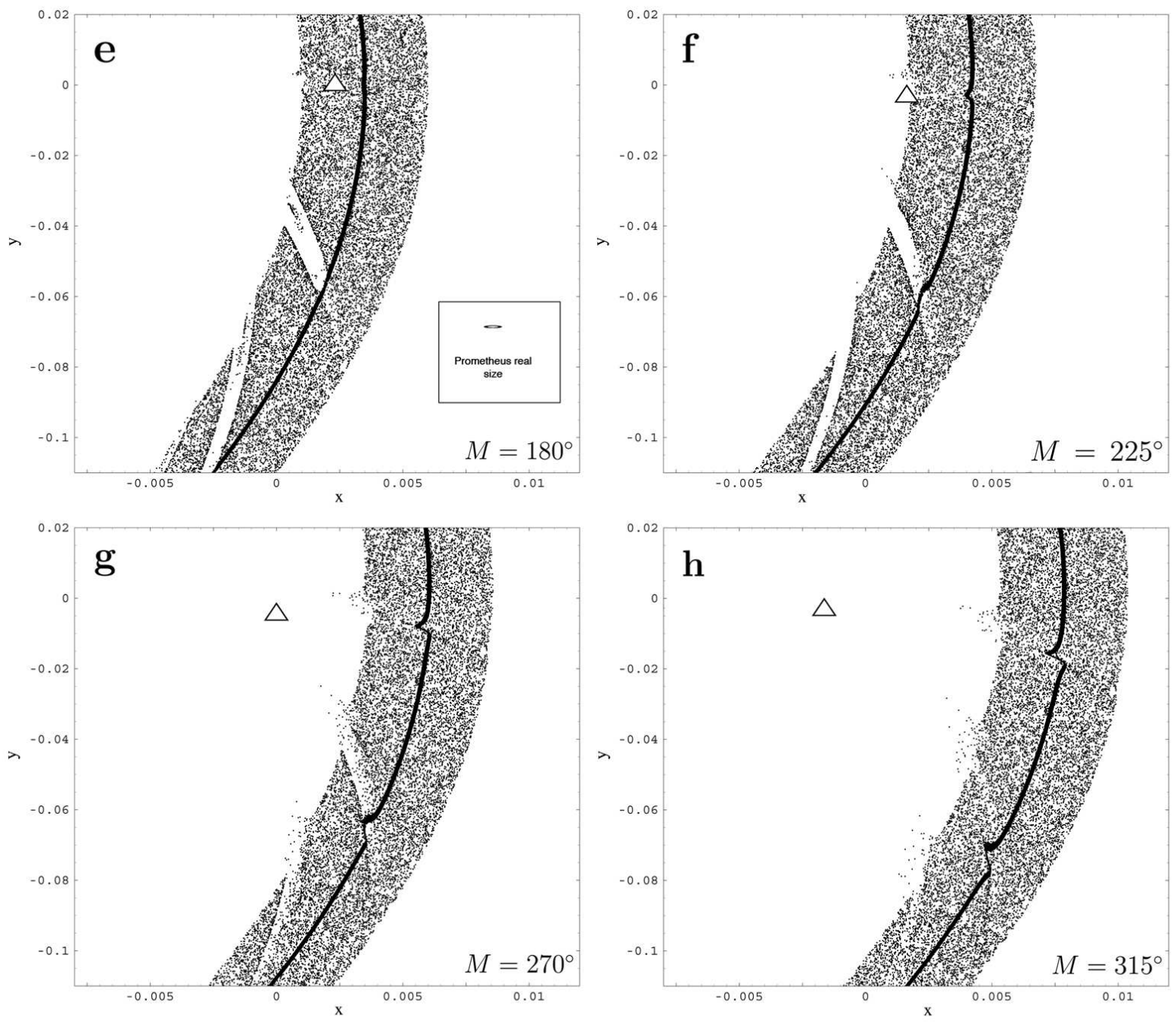

Fig. 3. Last four snapshots of the encounter between Prometheus and the F ring. Prometheus is represented as a triangle $(\triangle)$ and it is used to denote the position of the center of Prometheus, the true-scale of Prometheus is shown, as a elongated ellipse, in the left-bottom corner of Fig $3 \mathrm{k}$. These plots are in the reference frame that rotates with Prometheus mean motion. The mean anomaly $M$ for each of the plots is shown in the right bottom part of the plot. We observe that the ring is composed by two components: The core strand (at the center of the ring) and the background sheet (the less dense component that surrounds the core). 


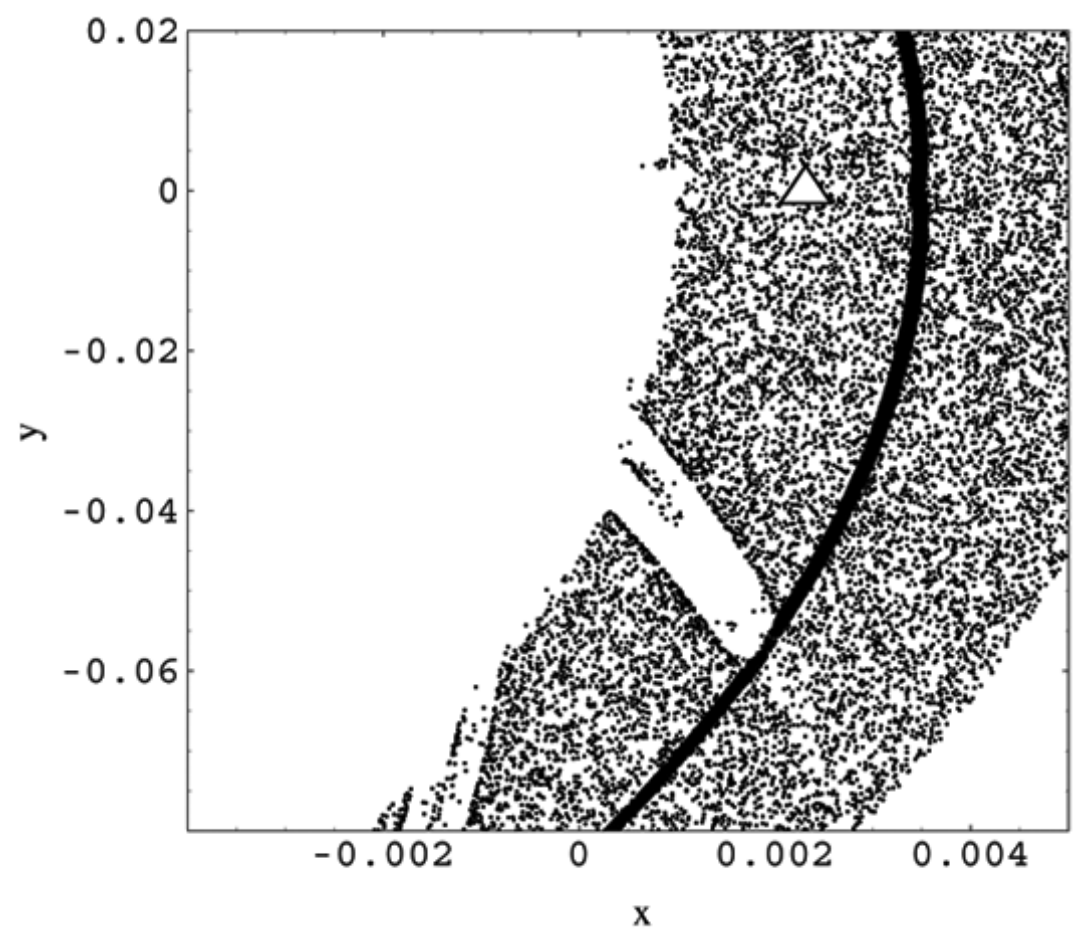

Fig. 4. Enlarged view of Fig. 3e. The mean anomaly of Prometheus is $M=180^{\circ}$. In this plot it is now clearer that this island is an accumulation of perturbed particles. This island appears only when Prometheus is close to apoasis (and the F ring to periapsis).

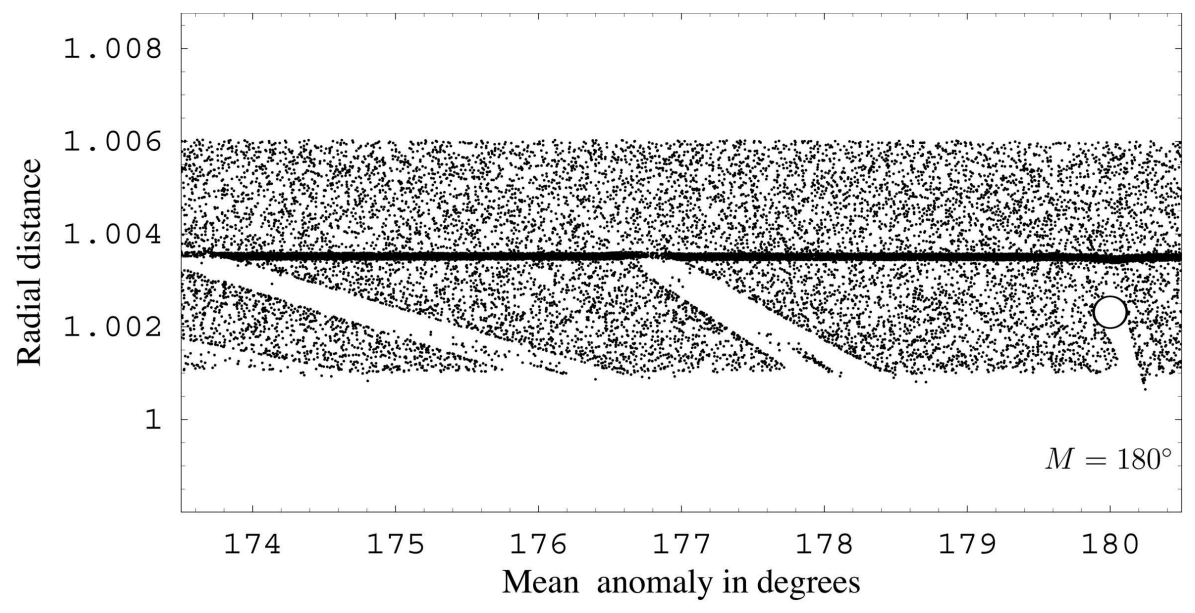

Fig. 5. Plot for the anti-alignment configuration with Prometheus' mean anomaly $M=180^{\circ}$. Prometheus, as can be seen in the plot, is at its deepest incursion into the ring. Notice the prominent channels. The islands in the middle of the channels are clearly visible too. 


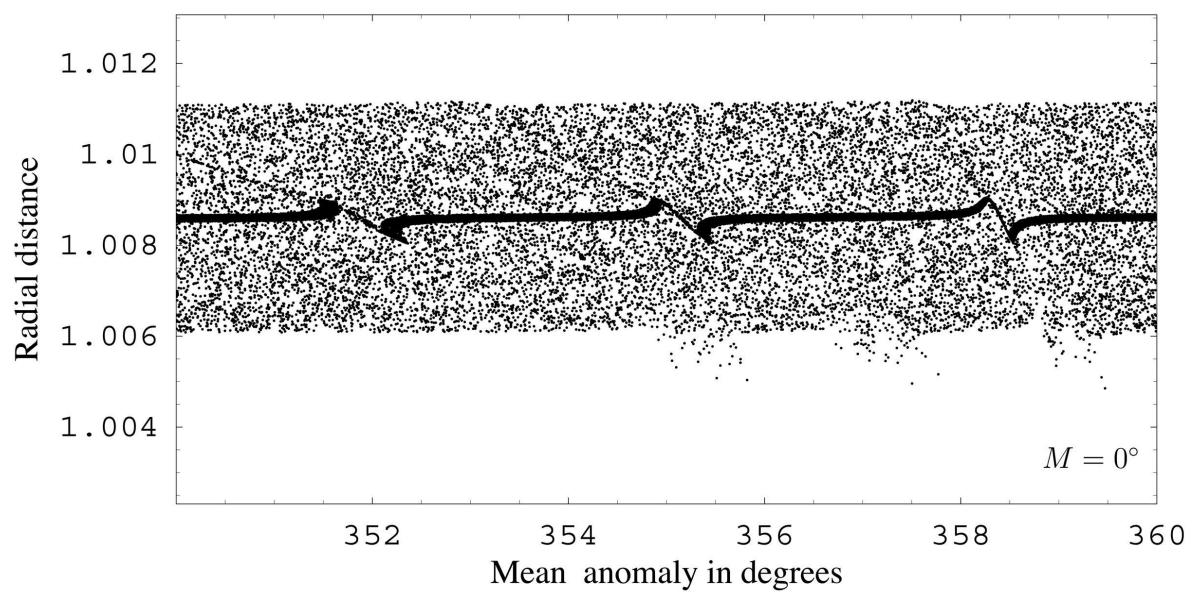

Fig. 6. Numerical model for the anti-alignment configuration, the mean anomaly of Prometheus is $M=0^{\circ}$. This is the possible appearance of the $\mathrm{F}$ ring for this configuration, Prometheus is at periapsis and the F ring is at apoapsis. As can be noticed the streamers are pronounced. Here Prometheus is too far away from the ring to be visible in the plot. 